\title{
The study on the LHAASO-WCDA time calibration
}

\author{
Jinyan Liu*', Bo Gao, Min Zha, Zhiguo Yao, Xiaojie Wang, for the LHAASO \\ Collaboration \\ Institute of High Energy Physics, CAS \\ E-mail: jyliudihep.ac.cn
}

\begin{abstract}
The Large High Altitude Air Shower Observatory (LHAASO) is a multi-purpose experiment, which is now under construction at Mt. Haizishan (4410 m a.s.l.), Daocheng, Sichun, China. The $78,000 \mathrm{~m}^{2}$ Water Cherenkov Detector Array (WCDA), one of main components of the LHAASO, can be able to survey the gamma-ray sky continuously in a wide energy range. The precision of the angular resolution is a key factor for the detection sensitivity of the gamma-ray sources. To reach the designed angular resolution, a time calibration system with a good accuracy is needed. A hardware calibration system based on optical fibers and blue LEDs have be deployed in WCDA, which provides initial time offsets for later analysis. Beside some other factors, such as PMT position differences and other unknown effects, will introduce additional time offsets between the detector cells. In order to calibrate such differences, an offline software calibration procedure based on the offline air shower data is suggested. In this paper, all above details in hardware and offline software calibration are introduced, some preliminary experimental result are also presented.
\end{abstract}

36th International Cosmic Ray Conference -ICRC2019-

July 24th - August 1st, 2019

Madison, WI, U.S.A.

\footnotetext{
* Speaker.

${ }^{\dagger}$ This work is supported in China by NSFC (11761141001, U1831208, 11705222, 11675187), the Chinese Ministry of Science and Technology, the Chinese Academy of Sciences, the Key Laboratory of Particle Astrophysics, IHEP, CAS.
} 


\section{Introduction}

The Large High Altitude Air Shower Observatory (LHAASO) [1] aims to investigate the evolution of the universe by studying the origin of high energy cosmic rays via the detection of air showers induced by gamma rays or cosmic rays [2]. It is located in Southwest China, near Mt. Haizishan at an altitude of $4410 \mathrm{~m}$ above sea level, and is still under construction. It consists of three detectors: a 1-km² extensive air shower array (KM2A), a water Cherenkov detector array (WCDA), and a wide field-of-view Cherenkov telescope array (WFCTA).

As one of the major detectors, the WCDA covers an area of 78,000 $\mathrm{m}^{2}$ and consists of three water pools, which are partitioned into 3120 detector cells in total, as shown in Fig. 1. The size of two pools is $150 \mathrm{~m} \times 150 \mathrm{~m}$ each, and that of the third one is $300 \mathrm{~m} \times 110 \mathrm{~m}$. Each cell is equipped with a large photomultiplier tube (PMT) with the photocathode facing upward to detect Cherenkov photons produced by secondary particles from cosmic ray showers in water [3]. The physics goal of the WCDA is to survey the northern sky for very-high-energy gamma ray sources. The accurate calibration of the detector is important to achieve a good spectrum measurement of the gamma ray sources [4].

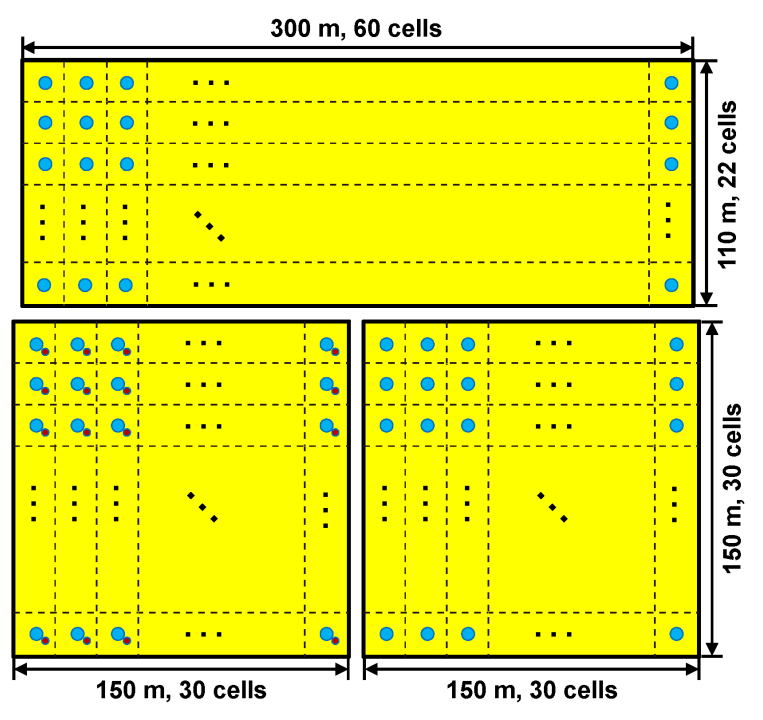

Figure 1: Schematic view of WCDA detector

For the WCDA, a primary particle is reconstructed from the shower front, based on the arrival time of the detected Cherenkov photons produced by the shower when passing through the water pool. The precision of reconstructed direction depends on the time accuracy and time resolution of the fired PMT channels. The time resolution is the statistical fluctuation of each detector cell. The time accuracy indicates the relative time offsets among different detector cells, originated mainly from hardware inconsistency from PMTs, cables and electronics. Time offsets without correction directly result in low efficiency and worse resolution of the direction reconstruction. Non-zero time offsets exist in the very beginning of the experiment and may be varying due to the variation of temperature during the data taking. Therefore, a precise, frequent and stable time calibration method must be developed. 
The paper is organized as follows. This section gives an overview of LHAASO-WCDA detector and the requirements of the time calibration of the detector. In section 2, the overview of the whole time calibration system is introduced. Section 3 explains the LED and optical fiber based calibration method. Section 4 introduces the detailed time calibration method to calibrate the WCDA using cosmic rays. Section 5 shows the preliminary performance. Finally, section 6 presents the conclusion.

\section{Overview of time calibration system}

To calibrate the time offset between different cells, a modularized calibration system is developed for the WCDA [5]. The WCDA is divided into clusters with $6 \times 6$ detector cells. To calibrate an inner cluster, a module equipped with a LED and 40 fibers is installed in the cluster. These detector cells are connected to 36 of the 40 fibers, while the remaining 4 fibers are reserved for backup. The LED is driven by a narrow pulse generator and then the signals are split into these fibers. The module itself is calibrated before being installed into the detector, so that it can calibrate the time offsets between these cells in the same cluster. In order to calibrate the adjacent clusters, other modules are installed that are connected by 20 fibers to the PMTs in the same cluster, while 16 fibers are connected to the PMTs in the adjacent clusters. By combining the results of the intraand inter-cluster modules, the time offset of the whole detector cells are calibrated.

However, the calibration system itself is not easy to be calibrated during the operation. For example, the environment temperature will affect the fibers in the calibration modules, which has impact on the performance of the calibration system. A solution is to use extensive air showers (EAS) events to calibrate the time offsets between all detector cells, a method that was adopted by several experiments such as ARGO-YBJ [6, 7], Tibet AS- $\gamma$ [8] and HAWC [9]. For WCDA scale detector arrays, all cells can be fired only with very-high-energy events. However, the flux of such events is very low. Hence only rare events can be used for time offset calibration of all cells at once.

To calibrate the detector frequently, a method using a high-statistics sample of low-energy cosmic rays is developed. Cosmic rays are one of major backgrounds for the WCDA. Compared with gamma rays, cosmic rays such as protons will produce many muons in the air showers. When these muons pass through water, Cherenkov photons are produced and detected by the PMTs. Due to the higher Cherenkov photon yield, cosmic rays can be distinguished from gamma rays. A cluster can be triggered by these low energy cosmic ray events, which are then reconstructed and used for time calibration. When accumulating enough events for one cluster, the time offsets in the cluster can be calibrated. The intra-clusters are calibrated first, then the adjacent clusters are calibrated using events that hit more than one cluster.

\section{LED and optical fiber based time calibration}

Neighbouring detector cells are partitioned by black plastic curtains to prevent the cross-talk of Cherenkov photons, causing difficulty on calibrating the whole array with a single light source. To addressing this problem, a cross-cluster calibration scheme is particularly designed for the WCDA detector. 
In accordance with the layout of the WCDA electronics system, the WCDA detector cells are grouped into clusters, each cluster containing $6 \times 6=36$ neighbouring cells [10]. Two sets of light sources - each consists of a blue LED, a light guide and a bundle of plastic optical fibers, are employed in a cluster, named module $A$ and module $B$, respectively. A module contains 40 optical fibers, among which only 36 are in use, and the remaining four are merely for backup.

Fig. 2 shows the schematic of the deployment of the fiber bundles of two neighboring clusters. In a particular cluster of the two, as for module $A$, all the 36 fibers in use run into the surface of PMTs inside the cluster; as for module $B$, however, four fibers are cross-linked to the PMTs in the other cluster, and the rest fibers remain in their own side. Given that an array contains tens of clusters and depending on the location, a cluster can have a maximum of four neighbours; hence, module B contains 4, 8, 12 or 16 fibers in a cluster cross-linked onto PMTs in other neighbouring clusters.

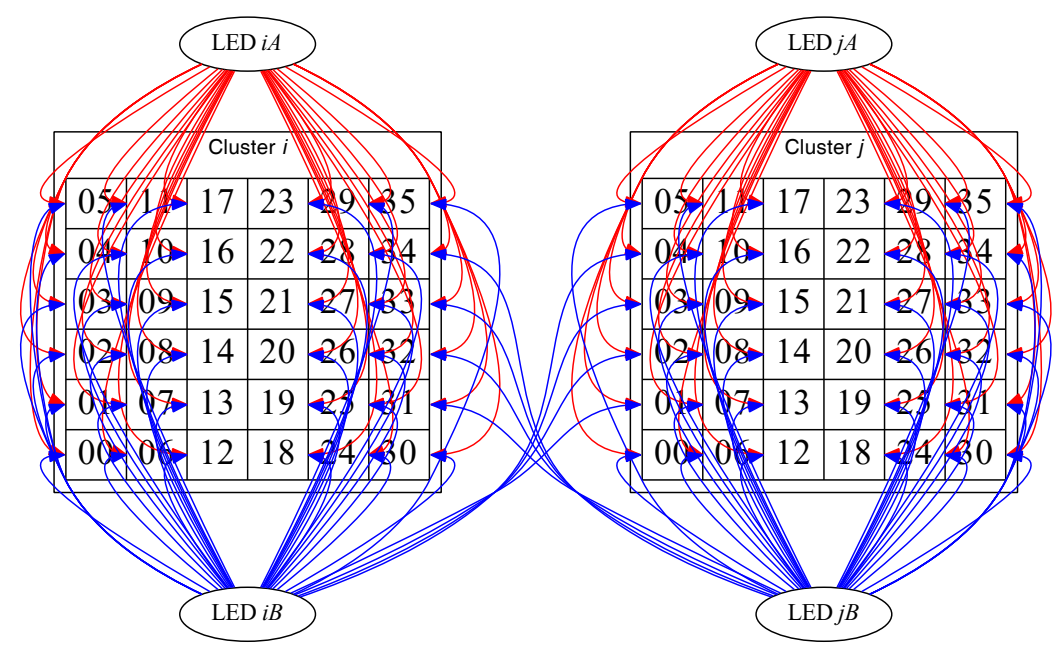

Figure 2: Schematic of cross-cluster fiber connections for two neighboring clusters. For visual clarity of the drawing, the exchange of fibers in module $B$ with more other clusters is not shown.

Every LED is driven by narrow voltage pulses with width around $10 \mathrm{~ns}$ generated by a simple electronic board, whose the frequency and amplitude are controlled by a remote system communicated via a fiber link. During the operation of the array, the two LEDs in a cluster are pulsed in turn and continuously. The tunable pulsation frequencies are generally low, e.g. 5-10 Hz, in order to avoid influence on interested physical signals.

The length of fibers differ, resulting in intrinsic time difference of LED lights traveling through fibers - that should be measured and registered before the installation.

During the operation of the array, the time difference of the PMT signals generated by the time calibration system in a cluster can be measured considering the intrinsic time difference of fibers to obtain the time offsets among the PMTs in addition to their backend hardware. With the above setting of the fibers, module $A$ calibrates the time offsets internally in a cluster, and module $B$ can combine the time offsets among neighbouring clusters. The whole array is then calibrated through propagating the time offsets from the array center outwards the sides with help of crossing fibers. That's the manner of working of the time calibration system. 


\section{Air shower data based offline time calibration}

The hardware method introduced in the last section can calibrate the whole detector cells. However, there are some effects not considered in this hardware method. The positions of PMTs are not considered, which can cause systematic time offsets in the final result. The varying temperature can also cause the time offsets. If there are some abnormal in the hardware calibration system, it is also difficult to calibrate the time offsets. Hence, an offline time calibration method is developed for WCDA.

A traditional calibration method is using the reconstructed events to calculate the residual time of each cell. The calibration of the detector such as WCDA will depend on rare very high energy events, which need a long term data taking to accumulate such events. In order to calibrate frequently, the detector is divided into overlapped clusters, which are calibrated using the high statistics low energy events.

The time calibration depends on the reconstruction of low energy cosmic rays. For low energy events, the propagation of cosmic rays can be described using a plane front, as shown in Fig. 3.

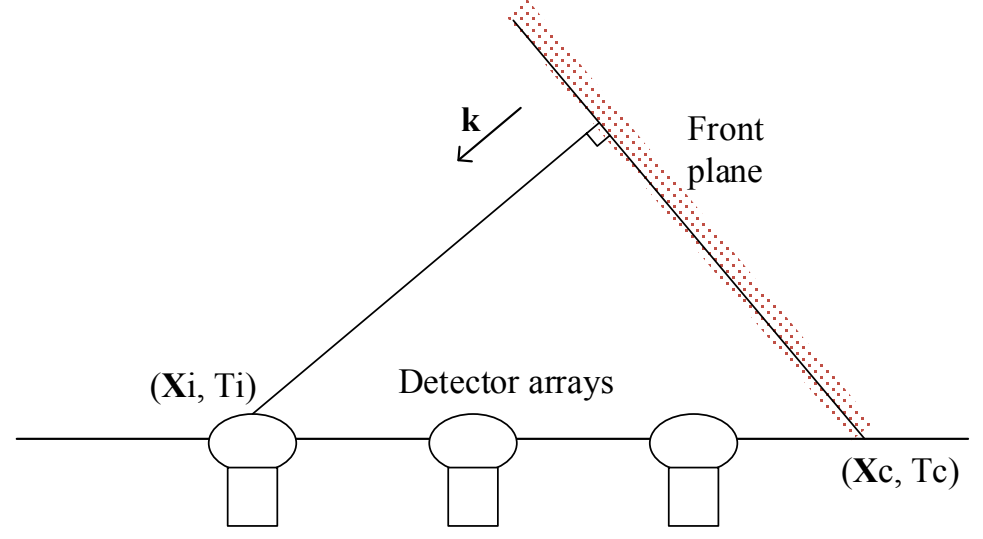

Figure 3: Schematic view of plane fit

Assuming at time $T_{c}$, the secondaries are at positions $\mathbf{X}_{c}$ in the plane front, which is represented as a vector. In the ground, the position of $i$-th PMT is vector $\mathbf{X}_{i}$. After propagation with light speed $c$, the secondaries hit the $i$-th PMT at time $T_{i}$. The ideal plane front equation is described as follows:

$$
T_{i}=T_{c}+\mathbf{k} \cdot\left(\mathbf{X}_{i}-\mathbf{X}_{c}\right) / c
$$

Hence, the predicated hit time $T_{i}^{\text {pred }}$ can be calculated using the plane front equation. A residual time between the measured hit time $T_{i}^{\text {meas }}$ and the predicated hit time can be defined as

$$
\delta_{i}=T_{i}^{\text {meas }}-T_{i}^{\text {pred }}
$$

Using all the fired PMTs, the direction of the cosmic ray $\mathbf{k}$ can be fitted by minimization of the residual time. Define $\chi^{2}$ as

$$
S=\chi^{2}=\sum_{i} \frac{\delta_{i}^{2}}{\sigma_{i}^{2}}=\sum_{i} \frac{\left(T_{i}^{\text {meas }}-T_{i}^{\text {pred }}\right)^{2}}{\sigma_{i}^{2}},
$$


where $\sigma_{i}$ is the error of $i$-th PMT.

Assuming that there is a systematic time offset $\Delta_{i}$ for the $i$-th PMT, which is not varying with the events. For an event $j$, the predicated hit time can be written as:

$$
T_{i j}^{\text {pred }}=T_{c j}+\mathbf{k}_{j} \cdot\left(\mathbf{X}_{i}-\mathbf{X}_{c j}\right) / c+\Delta_{i}
$$

The residual time of $i$-th PMT in event $j$ can be written:

$$
\delta_{i j}=T_{i j}^{\text {meas }}-T_{i j}^{\text {pred }}
$$

The $\chi^{2}$ of event $j$ is then defined as:

$$
\begin{aligned}
S_{j} & =\chi_{j}^{2}=\sum_{i} \frac{\delta_{i j}^{2}}{\sigma_{i j}^{2}} \\
& =\sum_{i} \frac{\left(T_{i j}^{\text {meas }}-\left(T_{c j}+\mathbf{k}_{j} \cdot\left(\mathbf{X}_{i}-\mathbf{X}_{c j}\right) / c+\Delta_{i}\right)\right)^{2}}{\sigma_{i j}^{2}},
\end{aligned}
$$

The sum of $\chi^{2}$ of all the events can be defined as:

$$
S=\sum_{j} S_{j}=\sum_{j} \chi_{j}^{2}
$$

To calibrate the time offset $\Delta_{i}$, the minimization of $\chi^{2}$ can be performed. The gradient descent algorithm can be used to get the time offset $\Delta_{i}$, which is written as the iteration of the $\Delta_{i}$ :

$$
\Delta_{i, n+1}=\Delta_{i, n}-\eta \frac{\partial S}{\partial \Delta_{i, n}}
$$

where $\eta$ is small enough. The gradient $\frac{\partial S}{\partial \Delta_{i}}$ is

$$
\begin{aligned}
\frac{\partial S}{\partial \Delta_{i}} & =-2 \sum_{j}\left(\frac{\delta_{i j}}{\sigma_{i j}^{2}}\right) \\
& =-2 \sum_{j}\left(\frac{T_{i j}^{\text {meas }}-\left(T_{c j}+\mathbf{k}_{j} \cdot\left(\mathbf{X}_{i}-\mathbf{X}_{c j}\right) / c+\Delta_{i}\right)}{\sigma_{i j}^{2}}\right)
\end{aligned}
$$

\section{Performance study}

To study on the performance of both calibration methods, two samples in the same day (May 31st, 2019) are selected. The first sample is the run of hardware calibration. Then after the hardware calibration run, the physics run is used as the second sample. The first sample could calibrate the time offsets with label \#0. Then they are used as the initial values for the software method. The second sample is used to calibrate the time offsets with label \#1. As figure 4 shown, the calibrated time offsets using two methods are consistent for most of the cells. 


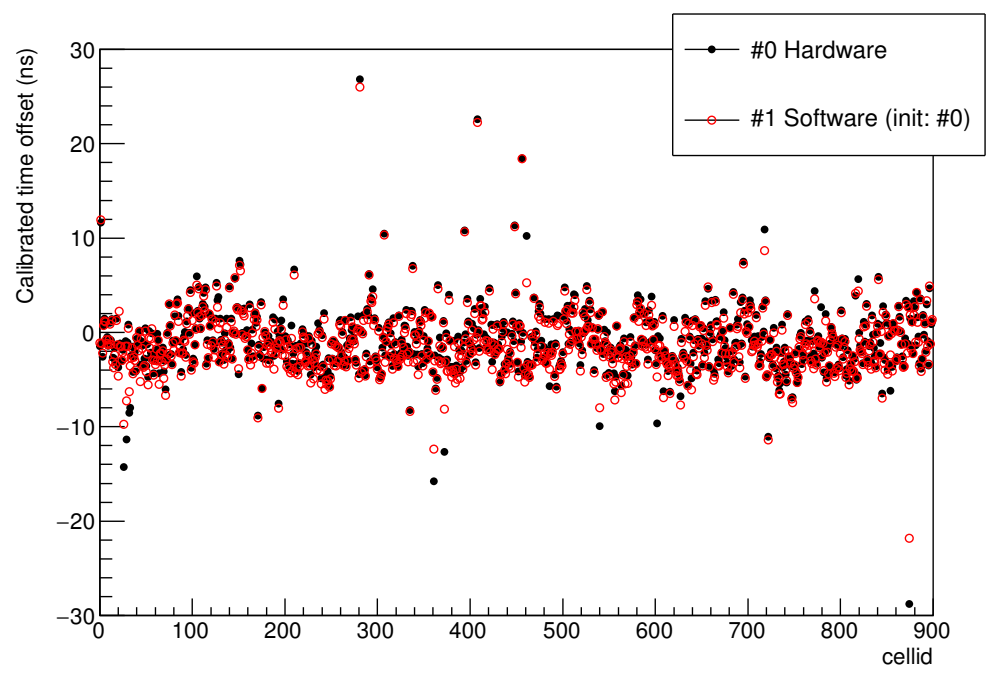

Figure 4: Calibrated time offsets

The differences between the two methods are then shown in figure. 5. As shown in the left figure, the time differences and positions are not correlated for most of detector cells. However, an additional correction in the future may be necessary at the detector edge. In the right figure, a gaussian function is used to fit the time difference. The sigma of gaussian function is $0.28 \pm 0.01 \mathrm{~ns}$.
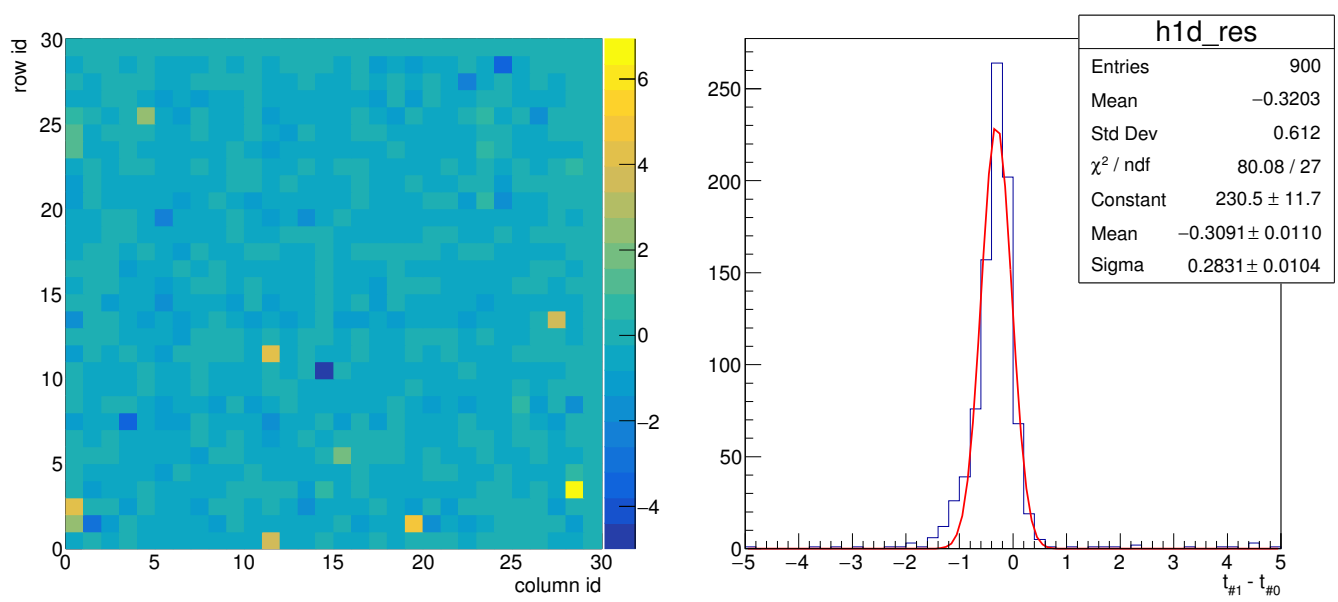

Figure 5: Comparison of calibrated time offsets between hardware method (\#0) and software method (\#1)

\section{Conclusion}

The 1/4 WCDA detector consisting 900 cells starts the data taking at the beginning of the year 2019. The LED and optical fiber based time calibration system is deployed in the detector, which calibrates the relative time offset every day. Then the hardware based calibrated time offsets are used as the input for the software method to get the final time offset. The preliminary results show 
that these calibrated time offsets using the two methods are consistent with each other. In this study, we only select the low energy physics events in a few minutes after the hardware calibration. In the future, the software method can be used to monitor the performance of the whole detector in real time.

\section{Acknowledgments}

This work is supported in China by NSFC (11761141001, U1831208, 11705222, 11675187), the Chinese Ministry of Science and Technology, the Chinese Academy of Sciences, the Key Laboratory of Particle Astrophysics, IHEP, CAS.

\section{References}

[1] Z. Cao,et al.,for the LHAASO Collaboration, A future project at tibet:the large high altitude air shower observatory(LHAASO), Chin. Phys. C (HEP \& NP) 34 (2010) pg.249-252

[2] S. Vernetto, Gamma Ray Astronomy with LHAASO, Journal of Physics 718 (2016) pg.052043 doi:10.1088/1742-6596/718/5/052043

[3] M.J. Chen, et al., for the LHAASO Collaboration, R\&D of LHAASO-WCDA, The 32th International Cosmic Ray Conference 9 (2011) pg.83 doi:10.7529/ICRC2011/V09/0732

[4] Z.G. YAO, et al., for the LHAASO Collaboration, Design and performance of LHAASO-WCDA experiment, The 32th International Cosmic Ray Conference 9 (2011) pg.95 doi:10.7529/ICRC2011/V09/0772

[5] J. Y. Liu et al., JINST 12, no. 10, P10021 (2017). doi:10.1088/1748-0221/12/10/P10021

[6] G. Aielli et al. [ARGO-YBJ Collaboration], Software timing calibration of the ARGO-YBJ detector, Astropart. Phys. 30 (2009) 287.

[7] M. Iacovacci et al. [ARGO-YBJ Collaboration], Time Calibration by Exploiting the Continuous Carpet Feature of ARGO-YBJ, in: Proceedings of the 33nd ICRC

[8] J. Huang et al. [Tibet ASgamma Collaboration], Measurement of high energy cosmic rays by the new Tibet hybrid experiment, PoS ICRC 2017 (2018) 484.

[9] H.A.Ayala Solares, et al., Timing Calibration of the HAWC Observatory, in: Proceedings of the 33rd ICRC 9 (2013) pg.95 doi:10.7529/ICRC2011/V09/0772

[10] L.Zhao, C.Ma, et al., Design of the readout electronics prototype for LHAASO WCDA ,in: Proceedings of the 20th IEEE-NPSS Real Time Conference, (2016) pg.3

doi:10.1109/RTC.2016.7543076 